\title{
EXISTENCIA DE SOLUCIONES DÉBILES PARA UNA CLASE DE ECUACIONES PARABÓLICAS NO LINEALES DEGENERADAS
}

\author{
Raúl Izaguirre Maguiña ${ }^{1}$, Julio Flores Dionición ${ }^{2}$ Victor Martínez León ${ }^{3}$, Eugenio Cabanillas \\ Lapa $^{4}$, Alfonso Perez Salvatierra ${ }^{5}$ \& Victor Carrera Barrantes ${ }^{6}$
}

Resumen: Sea $\Omega \subset \mathbb{R}^{n}$, un conjunto abierto, acotado con frontera regular $\Gamma$; $Q=\Omega \times(0, T)$ y $\Sigma=\Gamma \times(0, T)$, la frontera lateral del cilindro $Q$. Consideremos la siguiente ecuación parabólica no lineal de tipo degenerado:

$$
(*) \begin{cases}\frac{d}{d t}|u|^{\alpha} u-\operatorname{div}(k(\nabla u) \nabla u)+|u|^{\gamma} u=0 & \text { en } Q \\ u=0 & \text { en } \Sigma \\ u(0)=u_{0} & \text { en } \Omega\end{cases}
$$

Donde, $k: \mathbb{R}^{n} \rightarrow \mathbb{R}^{+} \cup\{0\}$, es una función lineal, continua $0<\alpha \leq 1,0<\beta$.

En este trabajo probamos la existencia de soluciones débiles para el problema $(*)$.

Palabras clave: Ecuaciones Diferenciales Parciales no Lineales, Ecuaciones Parabólicas Degeneradas, Método de Compacidad, Método de Galerkin.

\section{EXISTENCE OF WEAK SOLUTIONS FOR A TYPE OF DEGENERATE NONLINEAR PARABOLIC EQUATION}

Abstract: Let $\Omega \subset \mathbb{R}^{n}$, an open set, bounded with regular boundary $\Gamma ; Q=\Omega \times(0, T)$ and $\Sigma=\Gamma \times(0, T)$, the lateral boundary of the cylinder $Q$. Consider the following nonlinear parabolic equation of degenerate type:

$$
\text { (*) } \begin{cases}\frac{d}{d t}|u|^{\alpha} u-\operatorname{div}(k(\nabla u) \nabla u)+|u|^{\gamma} u=0 & \text { in } Q \\ u=0 & \text { in } \Sigma \\ u(0)=u_{0} & \text { in } \Omega\end{cases}
$$

Where, $k: \mathbb{R}^{n} \rightarrow \mathbb{R}^{+} \cup\{0\}$, is a continuous nonlinear function $0<\alpha \leq 1,0<\beta$. In this paper we show the existence of weak solution of the problem $(*)$

Key words: Nonlinear Partial Differential Equations, Degenerate Parabolic Equations, Compactness Method, Galerkin Method.

\footnotetext{
${ }^{1}$ UNMSM, Facultad de Ciencias Matemáticas, e-mail: raul_izaguirre2222@yahoo.es

${ }^{2}$ UNMSM, Facultad de Ciencias Matemáticas, e-mail: juliofloresd@hotmail.com

${ }^{3}$ UNMSM, Facultad de Ciencias Matemáticas, e-mail: vicaml19@gmail.com

${ }^{4}$ UNMSM, Facultad de Ciencias Matemáticas, e-mail: cleugenio@yahoo.com

${ }^{5}$ UNMSM, Facultad de Ciencias Matemáticas, e-mail: apersal@hotmail.com

${ }^{6}$ UNMSM, Facultad de Ciencias Matemáticas, e-mail: vcarrerab @yahoo.com
} 


\section{Introducción}

(En esta primera parte seguimos a Showalter R. [15])

Sea $\Omega$ un abierto de $\mathbb{R}^{n}$, con frontera $\Gamma$; $Q$ el cilindro $\Omega \times(0, T), 0<T<\infty$, con frontera lateral $\Sigma$ que representa un medio poroso, ocupado por un fluido (líquido o gaseoso) y este fluido se difunde desde lugares de mayor a menor presión. Sea $p(x, t)$ denota la presión del fluido en el punto $x \in \Omega$, y tiempo $t>0$ y denotamos la correspondiente densidad por $\rho(x, t)$. La cantidad de fluido en un elemento de volumen $\Omega_{0} \subset \Omega$ es $\int_{\Omega_{0}} c(x) \rho(x, t) d x$, y este define la porosidad del medio en el punto $x$. Esta es la fracción del volumen del medio accesible al fluido. El fluido es la tasa del fluido vectorial $\vec{J}(x, t)$, luego la tasa por la cual el fluido se desplaza atravesando un elemento de superficie $\Gamma_{0}$ con vector normal $\vec{\nu}$, está dado por $\int_{\Gamma_{0}} \vec{J}(x, t) \cdot \vec{\nu} d \Gamma$. Entonces la Ley de Consevación de fluidos aporta la forma integral:

$$
\frac{\partial}{\partial t} \int_{\Omega_{0}} c(x, t) \rho(x, t) d x+\int_{\Gamma_{0}} \vec{J} . \vec{\nu} d \Gamma=\int_{\Omega_{0}} f(x, t) d x, \quad \Omega_{0} \subset \Omega, \Gamma_{0}=\partial \Omega_{0}
$$

En la cual $f(x, t)$ denota cualquier fuente de densidad distribuida. .

Suponiendo que el flujo y la densidad son diferenciables, se puede escribir esto, en la forma diferencial:

$$
\frac{\partial}{\partial t} c(x) \rho(x, t)+\vec{\nabla} \cdot \vec{J}(x, t)=f(x, t), \quad x \in \Omega
$$

Según la Ley de Darcy's, el flujo depende del gradiente de la presión. Una de las formas de esta dependencia es:

$$
\vec{J}(x, t)=-\frac{k(x)}{\mu} \rho \vec{\nabla} p(x, t)
$$

Esto define la permeabilidad $k(x)$ del medio poroso. El valor de $k$ es una medida de la facilidad con la cual el fluido fluye a través del medio y $\mu$ es la viscosidad del fluido. Esto es, $1 / k$ es la resistencia del medio al flujo y $\mu$ es una correspondiente propiedad del fluido. Finalmente, el tipo de fluido considerado es descrito por la ecuación de estado:

$$
\rho=s(p)
$$

La función $s($.$) que relaciona la densidad y la presión es monótona. En efecto, esta es usualmente$ seleccionada como estrictamente creciente.

Reemplazando las cantidades apropiadas anteriores obtenemos:

$$
\frac{\partial}{\partial t} c(x) s(p(x, t))-\vec{\nabla} \cdot \frac{k(x)}{\mu} \vec{\nabla} S(p(x, t))=f(x, t), \quad x \in \Omega, t>0
$$

Donde $S(p)=\int_{0}^{p} s(\varepsilon) d \varepsilon$. Introduciendo la variable $u=S(p)$, obtenemos la ecuación general del medio poroso:

$$
\frac{\partial}{\partial t} c(x) a(u(x, t))-\vec{\nabla} \cdot \frac{k(x)}{\mu} \vec{\nabla} u(p(x, t))=f(x, t), \quad x \in \Omega, t>0
$$

Con $a(.) \equiv s(.) \circ S^{-1}($.

El caso clásico es obtenido seleccionando una ecuación de estado específico al flujo de gas. Esto corresponde a la selección de la función

$$
s(p)=\rho_{0} p^{\alpha}
$$


En la ecuación de estado, donde $\rho_{0}$ y $\alpha$ son constantes positivas con $\alpha \leq 1$. Si el medio es homogéneo, luego la porosidad y la permeabilidad son independientes de $x \in \Omega$, entonces esto lleva a la ecuación clásica del medio poroso

$$
\frac{\partial}{\partial t} c \rho-\frac{k}{\mu(\alpha+1) \rho_{0}^{m-1}} \Delta \rho^{m}=f
$$

Donde $m=1+\alpha^{-1}$. Otras situaciones en las cuales esta ecuación aparece incluye teoría de placas de frontera, donde $m=2$, modelos de poblaciones $(m>1)$ y en ciertos problemas de física del plasma $(0<m)$. La situación $m>1$, es llamada de difusión lenta, y el caso $0<m<1$, de difusión rápida. En el primer caso disturbios tienen velocidad de propagación finita, contrario al caso $m=1$ y cualquier solución que se inicie con soporte compacto continua teniendo soporte compacto para todo tiempo posterior.

La más simple situación es la de un fluido tenuemente compresible. Aquí la ecuación de estado tiene la forma $s(p)=\exp c_{0} p$, donde $c_{0}>0$, es la compresibilidad del fluido. Entonces la ecuación (.) se simplifica a la ecuación parabólica lineal

$$
\frac{\partial}{\partial t} c \rho-\frac{k c_{0}}{\mu} \Delta \rho=f
$$

Esta es formamente la misma que la ecuación del calor, esto es (.) con $m=1$.

Si suponemos que la ley de Darcy's tiene la forma no lineal

$$
\vec{J}=\frac{k(\rho \vec{\nabla} p)}{\mu} \rho \vec{\nabla} p
$$

En la cual la permeabilidad depende sobre el flujo, entonces en términos de la variable $u=S(p)$, obtenemos la ecuación del medio poroso casi lineal

$$
\frac{\partial}{\partial t} c(x) a(u(x, t))-\vec{\nabla} \cdot \frac{k(\vec{\nabla} u(x, t))}{\mu} \vec{\nabla} u(x, t)=f(x, t), \quad x \in \Omega, t>0
$$

Con $a(.) \equiv s(.) \circ S^{-1}($.$) , como antes.$

Finalmente si especializamos esto al caso de un fluido tenuemente compresible, obtenemos:

$$
\frac{\partial}{\partial t} c(x) c_{0} u(x, t)-\vec{\nabla} \cdot \frac{k(\vec{\nabla} u(x, t))}{\mu} \vec{\nabla} u(x, t)=f(x, t), \quad x \in \Omega, t>0
$$

Ecuaciones relacionadas con $(*)$ son por ejemplo:

$$
\begin{array}{ll}
\frac{d}{d t}\left|u^{\prime}\right|^{\alpha} u^{\prime}-\Delta u+|u|^{\rho} u=0 & \alpha, \rho>0 \\
\frac{d}{d t}|u|^{\alpha} u-\sum_{i=1}^{n} \frac{\partial}{\partial x_{i}}\left(\left|\frac{\partial u}{\partial x_{i}}\right|^{\beta} \frac{\partial u}{\partial x_{i}}\right)+b(x)|u|^{\rho} u=f & \alpha, \beta, \rho>0 \\
\lambda(x) u^{\prime}-\Delta\left(|u|^{\beta} u\right)-u^{p}=0 & \beta, \rho>0 \\
u^{\prime}-\nabla\left(u^{\beta} \nabla u\right)-u^{p}=0 & \beta, \rho>0 \\
\frac{d}{d t}|u|^{\alpha} u-\Delta|u|^{\beta} u+b(x)|u|^{\rho} u=f & \alpha, \beta, \rho>0
\end{array}
$$

que son tratadas en las referencias [2], [3], [4], [5], [12], [18] entre otras.

En relación a esta última ecuación, observamos que en el caso $\alpha=0, \beta=0,(*)$ representa una típica ecuación parabólica no lineal que ha ido bastante estudiada desde diversos puntos de vista 
en matemática pura y aplicada. Si $\alpha=0, \beta>0$ y $b(x)=1$, el problema $(*)$ aparece en el estudio de fenómenos de propagación del calos con conductividad térmica y fuentes de calor que dependen no linealmente de la temperatura del medio. En este caso resultados de existencia y no existencia de solución global para diversas posibilidades de los exponentes $\beta, \rho$ han sido obtenidos entre otros por Samarskii [12], [13], Ladyzhenskaya-Solonnikov-Urel'Teva [9], Galaktionov [3], [4], [5] y [6]. En particular en [5] se obtienen resultados de existencia global para $0<\beta<\rho, b(x)=-1$, usando para ello la técnica del "Potencial Well" introducida por Sattinger [13], para obtener solución global para ecuaciones de tipo hiperbólico con energía no positiva. En izaguirre [7],se demuestra existencia global para $\alpha \geq 0,0<\beta<\rho, b(x)=-1$.

En este trabajo determinamos condiciones para la existencia global de solución débil del problema $(*)$, para el caso $0 \leq \alpha \leq 1$ y los exponentes $\beta, \rho$ satisfaciendo condiciones tecnicas.

\section{Preliminares}

Sea $\Omega$ un abierto acotado de $\mathbb{R}^{n}$. Consideremos el espacio de Sobolev:

$$
W^{1, p}(\Omega)=\left\{u \in L^{p}(\Omega) ; \frac{\partial u}{\partial x_{i}} \in L^{p}(\Omega) \text { en el sentido de las distribuciones }\right\}
$$

Que es un espacio de Banach, con norma

$$
\|u\|=\left(|u|_{L^{p}(\Omega)}^{p}+\sum_{i=1}^{n}\left|\frac{\partial u}{\partial x_{i}}\right|_{L^{p}(\Omega)}^{p}\right)^{1 / p}
$$

y, $W_{0}^{i, p}(\Omega)$, la clausura en $W^{1, p}(\Omega)$, de $C_{0}^{\infty}(\Omega)$, el espacio de las funciones infinitamente diferenciables en $\Omega$, con soporte compacto. Este espacio es de Banach, con la norma

$$
\|u\|=\left(\sum_{i=1}^{n}\left|\frac{\partial u}{\partial x_{i}}\right|_{L^{p}(\Omega)}^{p}\right)^{1 / p}
$$

Sea $T>0$ un número real y $X$ un espacio de Banach, representaremos por $Z=L^{p}(0, T ; X)$, $1 \leq p \leq \infty$, el espacio de Banach de funciones (clases), $u:(0, T) \rightarrow X$, que son medibles y $\|u(x)\|_{X} \in L^{p}(0, T)$, con norma

$$
\|u\|_{L^{p}(0, T ; X)}=\left(\int_{0}^{T}\|u(t)\|_{X}^{p} d t\right), 1 \leq p<\infty
$$

y $\|u\|_{L^{\infty}(0, T ; X)}=\quad$ sup.ess $\|u(t)\|_{X}$. Luego $Z^{*}=L^{q}\left(0, T ; X^{*}\right)$, donde $p^{-1}+q^{-1}=1$. Si $2 \leq p<\infty$, entonces $q \leq p$ y por lo tanto $Z \subset Z^{*}$. También con $h_{\gamma}$, denotamos la función real

$$
h_{\gamma}(s)=|s|^{\gamma} s, \quad \gamma>-1 \text {. }
$$

Lema 2.1. Sea $\alpha \in(-1, \infty)$ y la función, $h_{\alpha}: \mathbb{R} \rightarrow \mathbb{R}$, definida por, $h_{\alpha}(s)=|s|^{\alpha}$ s. Entonces

$h_{\alpha}$ es una función monótona, estrictamente creciente.

$h_{\alpha}$ es derivable, con función derivada $h_{\alpha}^{\prime}(s)=(\alpha+1)|s|^{\alpha}$.

$h_{\alpha}^{-1}(s)=h_{\frac{-\alpha}{\alpha+1}}(s)$.

La aplicación $h_{\alpha}: L^{q}(\Omega) \rightarrow L^{q /(\alpha+1)}(\Omega)$, es continua, para $q \geq \alpha+1$. 
Demostración. (Ver Izaguirre [7]).

Lema 2.2. Sea $k: \mathbb{R}^{n} \rightarrow \mathbb{R}$, una función continua, con $k(0)=0$,definimos $f(\xi)=k(\xi) \xi$, $L(\xi)=k(\xi)|\xi|^{2}, \xi \in \mathbb{R}^{n}$, entonces:

$$
\nabla L(\xi)=k(\xi) \xi ; \xi \in \mathbb{R}^{n}
$$

Si $L$ es convexa, entonces $f$ es monótona.

Demostración. (Ver Berger [1]).

Lema 2.3. Sean $X, Y, Z$ espacios de Banach tales que $X \subset Y \subseteq Z$, con inmersiones continuas y la inmersión $X \subset Y$ es compacta. Sea

$$
W=\left\{u \in L^{p}(0, T ; X) ; u^{\prime} \in L^{q}(0, T ; Z)\right\}
$$

donde $u^{\prime}$ denota la derivada generalizada de $u:[0, T] \rightarrow X$, sobre $(0, T)$. Entonces

$$
\begin{aligned}
& \text { Si } p=\infty, q>1 \text { entonces } W \subseteq C([0, T] ; Z) \text { es compacta. } \\
& \text { Si } 1 \leq p<\infty, q=1 \text { entonces } W \subseteq L^{q}(0, T ; Y) \text { es compacta. }
\end{aligned}
$$

Demostración. (Ver J. Simon [16]).

Teorema 2.1. (Sobolev). Sea $\Omega$, un conjunto abierto, acotado y regular de $\mathbb{R}^{n}, 1 \leq p<\infty$. Entonces:

$$
\begin{aligned}
& \text { Si, } p<n, W_{0}^{1, p}(\Omega) \subset L^{q}(\Omega) ; p \leq q \leq \frac{n p}{n-p}=p^{*} \\
& \text { Si, } p=n, W_{0}^{1, p}(\Omega) \subset L^{q}(\Omega) ; p \leq q<\infty \\
& \text { Si, } p>n, W_{0}^{1, p}(\Omega) \subset C^{0}(\bar{\Omega})
\end{aligned}
$$

Demostración. (Ver Berger [1]).

HIPÓTESIS. Establecemos las siguientes condiciones para los parámetros $\alpha, \beta$ y sobre la función $k$ :

$(\mathrm{H}-1) 0<\alpha \leq 1$.

$(\mathrm{H}-2)-1<\gamma<\infty$.

$(\mathrm{H}-3) k: \mathbb{R}^{n} \rightarrow \mathbb{R}$, es una función continua y $k(0)=0$.

$(\mathrm{H}-4)$ Existen constantes positivas $C_{1}, C_{2}$, tales que:

$$
C_{1}|\xi|^{p-2} \leq k(\xi) \leq C_{2}|\xi|^{p-2}, \quad \xi \in \mathbb{R}^{n}, p \geq \alpha+2>2 .
$$

También consideramos los siguientes espacios funcionales:

$$
V=W_{0}^{1, p}(\Omega) \cap L^{\gamma+2}(\Omega)
$$

Entonces el espacio $V$, dotado con la norma

$$
|u|_{V}=|u|_{W_{0}^{1, p}(\Omega)}+|u|_{L^{\gamma+2}(\Omega)}, \quad u \in V
$$

Es un espacio de Banach, reflexivo y separable y se tiene que

$$
V^{\prime}=W^{-1, p^{\prime}}(\Omega)+L^{\gamma+2 /(\gamma+1)}(\Omega)
$$


EL OPERADOR A. Consideramos el operador diferencial:

$$
A(u)=-\nabla \cdot k(\nabla u) \nabla u=-\sum_{i=1}^{n} \frac{\partial}{\partial x_{i}}(k(\nabla u) \nabla u), \quad u \in W_{0}^{1, p}(\Omega)
$$

Entonces:

$$
A: L^{p}\left(0, T ; W_{0}^{1, p}(\Omega)\right) \rightarrow L^{p^{\prime}}\left(0, T ; W^{-1, p}(\Omega)\right), \frac{1}{p}+\frac{1}{p^{\prime}}=1
$$

es un operador monótono y hemicontinuo.

Entonces formulamos el teorema principal:

Teorema 2.2. Sea $T>0$ y $u_{0} \in V$. Entonces existe una función vectorial $u:[0, T] \rightarrow V$, tal que:

$$
\begin{gathered}
u \in L^{\infty}(0, T ; V) \\
A(u) \in L^{p^{\prime}}\left(0, T ; V^{\prime}\right)
\end{gathered}
$$

solución del problema:

$$
\begin{aligned}
& \left.h_{\alpha}(u)^{\prime}+A(u)+h_{\gamma}(u)=0, \text { en } L^{p^{\prime}} 0, T ; V^{\prime}\right) \\
& u(0)=u_{0}
\end{aligned}
$$

Demostración. Sea $\left(w_{j}\right)_{j \geq 1}$, una base de $V, V_{m}=\left[w_{1}, w_{2}, \ldots, w_{m}\right] \subset V$. Luego $V_{m}$ es un subespacio de $V$ de dimensión finita $m$.

Sea entonces:

$$
u_{m}(t)=\sum_{i=1}^{n} g_{i m}(t) w_{i} \in V_{m}
$$

Donde las funciones $g_{i m}$, son determinadas por la solución del siguiente sistema perturbado de ecuaciones diferenciales ordinarias no lineales:

$$
(\Delta)\left\{\begin{array}{l}
\left(h_{\alpha}\left(u_{\varepsilon m}(t)\right)^{\prime}, w_{j}\right)+\varepsilon\left(u_{\varepsilon m}(t), w_{j}\right)+\left(k\left(\nabla u_{\varepsilon m}(t)\right) \nabla u_{\varepsilon m}(t), \nabla w_{j}\right)+\left(h_{\gamma}\left(u_{\varepsilon m}(t)\right), w_{j}\right)=0 \\
\forall j=1,2, \ldots, m \\
u_{m}(0)=u_{0 m} \rightarrow u_{0} \text { en } V
\end{array}\right.
$$

El sistema es equivalente con el sistema no lineal de ecuaciones diferenciales ordinarias:

$$
(*)\left\{\begin{array}{l}
\vec{g}_{\varepsilon m}^{\prime}(t)=F\left(t, \vec{g}_{\varepsilon m}(t)\right) \\
\vec{g}_{\varepsilon m}(0)=\vec{g}_{0 m}
\end{array}\right.
$$

Donde:

$$
\begin{aligned}
& \left.F\left(t, \vec{g}_{\varepsilon m}(t)\right)=-C(t) B\left(t, \vec{g}_{\varepsilon m}(t)\right) \vec{g}_{\varepsilon m}(t)+H\left(\vec{g}_{\varepsilon m}(t)\right)\right) \\
& \vec{g}_{\varepsilon m}^{\perp}(t)=\left(g_{\varepsilon 1 m}, \ldots, g_{\varepsilon m m}\right) \\
& C(t)=A^{-1}, A(t)=\left(a_{i j}(t)\right), a_{i j}(t)=\left(\left|u_{\varepsilon m}\right|^{\alpha} w_{i}, w_{j}\right)+\varepsilon\left(w_{i}, w_{j}\right) \\
& B\left(t, \vec{g}_{\varepsilon m}(t)\right)=\left(g_{i j}\left(t, \vec{g}_{\varepsilon m}(t)\right)\right), g_{i j}\left(t, \vec{g}_{\varepsilon m}(t)\right)=\left(k\left(u_{\varepsilon m}(t)\right) \nabla w_{j}, \nabla w_{j}\right) \\
& H\left(\vec{g}_{\varepsilon m}(t)\right)^{\perp}=\left(\left(h_{\gamma}\left(u_{\varepsilon m}(t)\right), w_{1}\right), \ldots,\left(h_{\gamma}\left(u_{\varepsilon m}(t)\right), w_{m}\right)\right)
\end{aligned}
$$

En esta parte y para justificar la necesidad de perturbar el sistema, es necesario demostrar la existencia de la inversa de la matriz $A(t)$, lo cual se puede realizar de la siguiente forma: Sean $A_{j}(t)$, las columnas de la matriz $A(t)$ y supongamos que

$$
\sum_{j=1}^{m} \alpha_{j} A_{j}(t)=0
$$


Entonces:

$$
\sum_{j=1}^{n}\left(\left(\left|u_{\varepsilon m}(t)\right|^{\alpha}+\varepsilon\right) \alpha_{j} w_{j}, w_{i}\right)=0, \quad \forall i=1, \ldots, m
$$

Desde que $\left(w_{i}\right), i=1, \ldots, n$, es una base para $V_{m}$, tenemos que:

$$
\sum_{j=1}^{n}\left(\left|u_{\varepsilon m}(t)\right|^{\alpha}+\varepsilon\right) \alpha_{j} w_{j}=\left(\left|u_{\varepsilon m}(t)\right|^{\alpha}+\varepsilon\right) \sum_{j=0}^{n} \alpha_{j} w_{j}=0, \quad \forall i=1, \ldots, m
$$

De donde $\alpha_{j}=0, j=1, \ldots, m$. Luego la matriz $A(t)$, es invertible.

El sistema $(*)$ entonces, admite solución en un intervalo $\left[0, t_{\varepsilon m}\right)$, de donde seguimos la existencia de las soluciones aproximadas $u_{\varepsilon m}$ para $m \geq 1, \varepsilon>0 m \geq 1$. Seguidamente debemos obtener estimados a priori (acotaciones), para la sucesión $\left\{u_{\varepsilon m}\right\}$ de modo que podamos prolongarlas al intervalo $[0, T]$.

ESTIMADO A PRIORI 1. Por la linealidad, la igualdad $(\Delta)$ se verifica para todo $\nu \in V_{m}$. Entonces haciendo $\nu=u_{m}(t) \in V_{m}$, en la ecuación aproximada, obtenemos:

$$
\frac{d}{d t} \frac{1}{(\alpha+2)}\left|u_{\varepsilon m}(t)\right|_{\alpha+2}^{\alpha+2}+\frac{\varepsilon}{2} \frac{d}{d t}\left|u_{\varepsilon m}(t)\right|^{2}+\left|k^{1 / 2}\left(\nabla u_{\varepsilon m}(t)\right)\right|\left|\nabla u_{\varepsilon m}(t)\right|^{2}+\left|u_{\varepsilon m}(t)\right|_{\gamma+2}^{\gamma+2}=0
$$

integrando en la igualdad de 0 a $t$, obtenemos:

$$
\begin{aligned}
\frac{1}{(\alpha+2)}\left|u_{\varepsilon m}(t)\right|_{\alpha+2}^{\alpha+2}+ & \frac{\varepsilon}{2}\left|u_{\varepsilon m}(t)\right|^{2}+\int_{0}^{t}\left(\left|k^{1 / 2}\left(\nabla u_{\varepsilon m}(s)\right)\right|\left|\nabla u_{\varepsilon m}(s)\right|^{2}+\left|u_{\varepsilon m}(s)\right|_{\gamma+2}^{\gamma+2}\right) d s= \\
& =\frac{1}{(\alpha+2)}\left|u_{\varepsilon m}(0)\right|_{\alpha+2}^{\alpha+2}+\frac{\varepsilon}{2}\left|u_{\varepsilon m}(0)\right|^{2}=\frac{1}{(\alpha+2)}\left|u_{0 m}\right|_{\alpha+2}^{\alpha+2}+\frac{\varepsilon}{2}\left|u_{0 m}\right|^{2} \leq C
\end{aligned}
$$

Luego

$$
\begin{aligned}
& \left(u_{\varepsilon m}\right) \text { es acotada en } L^{p}\left(0, T ; W_{0}^{1, p}(\Omega)\right) \cap L^{\gamma+2}(Q) \\
& \left(\varepsilon^{1 / 2} u_{\varepsilon m}\right) \text { es acotada en } L^{\infty}\left(0, T ; L^{2}(\Omega)\right)
\end{aligned}
$$

ESTIMADO A PRIORI 2. Haciendo $\nu=u_{\varepsilon m}^{\prime}(t) \in V_{m}$, en la ecuación aproximada, obtenemos:

$$
\frac{2}{(\alpha+2)}\left|\frac{d}{d t} h_{\frac{\alpha}{2}}\left(u_{\varepsilon m}(t)\right)\right|^{2}+\frac{\varepsilon}{2}\left|u_{\varepsilon m}^{\prime}(t)\right|^{2}+\frac{d}{d t} L\left(\nabla u_{\varepsilon m}(t)\right)+\frac{1}{\gamma+2} \frac{d}{d t}\left|u_{\varepsilon m}(t)\right|_{\gamma+2}^{\gamma+2}=0
$$

integrando en esta igualdad:

$$
\begin{aligned}
\frac{2}{(\alpha+2)} \int_{0}^{t}\left|\frac{d}{d s} h_{\frac{\alpha}{2}}\left(u_{\varepsilon m}(s)\right)\right|^{2} d s+\frac{\varepsilon}{2} \int_{0}^{t}\left|u_{\varepsilon m}^{\prime}(s)\right|^{2} d s+L\left(\nabla u_{\varepsilon m}(t)\right)+\frac{1}{\gamma+2}\left|u_{\varepsilon m}(t)\right|_{\gamma+2}^{\gamma+2} & = \\
& =L\left(\nabla u_{0 m}\right)+\frac{1}{\gamma+2}\left|u_{0 m}\right|_{\gamma+2}^{\gamma+2} \leq C
\end{aligned}
$$

Luego:

$$
\begin{aligned}
& \frac{d}{d t}\left(h_{\frac{\rho}{2}}\left(u_{\varepsilon m}\right)\right) \text { es acotada en } L^{2}\left(0, T ; L^{2}(\Omega)\right) \\
& \left(\varepsilon^{1 / 2} u_{\varepsilon m}^{\prime}\right) \text { es acotada en } L^{2}\left(0, T ; L^{2}(\Omega)\right) \\
& \left(u_{\varepsilon m}\right) \text { es acotada en } L^{\infty}(0, T ; V)
\end{aligned}
$$


Con los estimados obtenidos demostraremos que:

$$
\left(h_{\alpha}\left(u_{\varepsilon m}\right)\right) \rightarrow h_{\alpha}(u) \text { débil en } L^{\infty}\left(0, T ; L^{p^{*} /(\alpha+1)}(\Omega)\right) \subset L^{\infty}\left(0, T ; L^{2 p^{*} /\left(p^{*}-\alpha\right)}(\Omega)\right)
$$

En efecto. Sea

$$
w_{\varepsilon m}=h_{\alpha / 2}\left(u_{\varepsilon m}\right)
$$

Entonces

$$
\begin{aligned}
& \left(w_{\varepsilon m}\right) \text { es acotada en } L^{\infty}\left(0, T ; W_{0}^{1, p / 2}(\Omega)\right) \\
& \left(w_{\varepsilon m}^{\prime}\right) \text { es acotada en } L^{2}\left(0, T ; L^{2}(\Omega)\right)
\end{aligned}
$$

Desde que, por el Teorema 2.1

$$
\begin{aligned}
& \left(u_{\varepsilon m}\right) \text { es acotada en } L^{\infty}\left(0, T ; L^{p^{*}}(\Omega)\right) ; p *=\frac{n p}{n-p} \geq p \\
& \left(\nabla u_{\varepsilon m}\right) \text { es acotada en } L^{\infty}\left(0, T ; L^{p}(\Omega)\right)
\end{aligned}
$$

Luego

$$
\nabla w_{\varepsilon m}=\frac{\alpha+2}{2}\left|w_{\varepsilon m}\right|^{\frac{\alpha}{2}} \nabla w_{\varepsilon m}
$$

entonces

$$
\int_{\Omega}\left|w_{\varepsilon m}\right|^{\alpha p / 4}\left|\nabla w_{\varepsilon m}\right|^{p / 2} d x \leq\left(\int_{\Omega}\left|w_{\varepsilon m}\right|^{\alpha p / 2}\right)^{1 / 2}\left(\int_{\Omega}\left|w_{\varepsilon m}\right|^{p}\right)^{1 / 2}<\infty
$$

Desde que, $0<\alpha \leq 1$, implica $\alpha p \leq 2 p \leq 2 p^{*}$.

Por resultados de inmersión sobre espacios de Sobolev, se tiene que:

$$
\begin{aligned}
& W^{1, p / 2}(\Omega) \subset L^{n p /(2 n-p)}(\Omega) \subset L^{2}(\Omega), n>p>\alpha+2 \\
& \text { La inmersión } W_{0}^{1, p / 2}(\Omega) \subset L^{n p /(2 n-p)}(\Omega) \text { es compacta. }
\end{aligned}
$$

Hacemos $\varepsilon=1 / m$. Entonces por el Lema 2.3, existe una subsucesión de $\left(u_{\varepsilon m}\right)$, que continuamos denotando de la misma forma, tal que:

$$
w_{\varepsilon m} \rightarrow w \text { fuerte en } C^{0}\left([0, T] ; L^{2}(\Omega)\right)
$$

Definimos $u$, tal que, $w=h_{(\alpha+1) / 2}(u)$. Entonces por continuidad:

$$
u_{\varepsilon m}=h_{\alpha / 2}^{-1}\left(w_{\varepsilon m}\right) \rightarrow u=h_{\alpha / 2}^{-1}(w) \text { fuerte en } C^{0}\left([0, T] ; L^{\alpha+2}(\Omega)\right)
$$

De donde:

$$
h_{\alpha}\left(u_{\varepsilon m}\right) \rightarrow h_{\alpha}(u) \text { fuerte en } C^{0}\left([0, T] ; L^{(\alpha+2) /(\alpha+1)}(\Omega)\right)
$$

Luego:

$$
\begin{gathered}
\left|u_{\varepsilon m}(0)\right|_{\alpha+2}^{\alpha+2} \rightarrow\left|u_{0}\right|_{\alpha+2}^{\alpha+2} \\
\left|u_{\varepsilon m}(T)\right|_{\alpha+2}^{\alpha+2} \rightarrow|u(T)|_{\alpha+2}^{\alpha+2}
\end{gathered}
$$

Asimismo

$$
h_{\gamma}\left(u_{\varepsilon m}\right) \rightarrow h_{\gamma}(u) \text { en casi todo punto de } Q
$$

y

$$
\left(h_{\gamma}\left(u_{\varepsilon m}\right)\right) \text { es acotada en } L^{\gamma+2 / \gamma+1}(Q) .
$$


Por (54), (55) y el Lema de Lions:

$$
h_{\gamma}\left(u_{\varepsilon m}\right) \rightarrow h_{\gamma}(u) \text { débil en } L^{\gamma+2 / \gamma+1}(Q)
$$

También obtenemos el siguiente estimado:

$$
\left(h_{\alpha}\left(u_{\varepsilon} m\right)^{\prime}\right) \text { es acotada en } L^{2 p^{*} /\left(p^{*}+\alpha\right)}\left(0, T ; L^{2 p^{*} /\left(p^{*}+\alpha\right)}(\Omega)\right) ; p^{*}=\frac{n p}{n-p} \geq p
$$

En efecto, desde la condición (2), y haciendo

$$
r=2 p^{*} /\left(p^{*}+\alpha\right), \theta=\left(p^{*}+\alpha\right) / \alpha, \theta^{\prime}=\left(p^{*}+\alpha\right) / p^{*}
$$

Observamos que:

$$
h_{\alpha}\left(u_{\varepsilon m}\right)^{\prime}=(\alpha+1)\left|u_{\varepsilon m}\right|^{\alpha} u_{\varepsilon m}^{\prime}=(\alpha+1)\left|u_{\varepsilon m}\right|^{\alpha / 2}\left|u_{\varepsilon m}\right|^{\alpha / 2} u_{\varepsilon m}^{\prime}=\frac{2(\alpha+1)}{(\alpha+2)}\left|u_{\varepsilon m}\right|^{\alpha / 2} h_{\alpha / 2}\left(u_{\varepsilon m}\right)^{\prime}
$$

Entonces:

$$
\int_{Q}\left|u_{\varepsilon m}\right|^{\alpha r / 2}\left|h_{\alpha / 2}\left(u_{\varepsilon m}\right)^{\prime}\right|^{r} d Q \leq\left(\int_{Q}\left|u_{\varepsilon m}\right|^{p^{*}} d Q\right)^{1 / \theta}\left(\int_{Q}\left|h_{\alpha / 2}\left(u_{\varepsilon m}\right)^{\prime}\right|^{2} d Q\right)^{1 / \theta^{\prime}}<\infty
$$

En conclusión, podemos extraer una subsucesión de $\left(u_{\varepsilon m}\right)$ que continuamos denotando de la misma forma tal que:

$$
\begin{array}{ll}
\left(u_{\varepsilon m}\right) \rightarrow u & \text { débil-* en } L^{\infty}(0, T ; V) \\
\left(\varepsilon^{1 / 2} u_{\varepsilon m}^{\prime}\right) \rightarrow 0 & \text { débil en } L^{2}\left(0, T ; L^{2}(\Omega)\right) \\
\left(h_{\alpha}\left(u_{\varepsilon m}\right)\right) \rightarrow h_{\alpha}(u) & \text { fuerte en } C^{0}\left([0, T] ; L^{(\alpha+2) /(\alpha+1)}(\Omega)\right) \\
\left(h_{\alpha}\left(u_{\varepsilon m}\right)^{\prime}\right) \rightarrow h_{\alpha}(u)^{\prime} & \text { débil en } L^{r}\left(0, T ; L^{r}(\Omega)\right) \\
\left(A\left(u_{\varepsilon m}\right)\right) \rightarrow \chi & \text { débil-* en } L^{p^{\prime}}\left(0, T ; V^{\prime}\right) \\
\left(h_{\gamma}\left(u_{\varepsilon m}\right)\right) \rightarrow h_{\gamma}(u) & \text { débil-* en } L^{\infty}\left(0, T ; L^{\gamma+2 / \gamma+1}(\Omega)\right)
\end{array}
$$

Entonces:

$$
\begin{gathered}
\int_{0}^{T}\left(h_{\alpha}\left(u_{\varepsilon m}\right)^{\prime}, w\right) \varphi(t) d t \rightarrow \int_{0}^{T}\left(h_{\alpha}(u)^{\prime}, w\right) \varphi(t) d t \\
\varepsilon \int_{0}^{T}\left(u^{\prime}, w\right) \varphi(t) d t=\sqrt{\varepsilon} \int_{0}^{T}\left(\sqrt{\varepsilon} u^{\prime}, w\right) \varphi(t) d t \rightarrow 0 \\
\int_{0}^{T}\left(A\left(u_{\varepsilon m}\right), w\right) \varphi(t) d t \rightarrow \int_{0}^{T}(\chi, w) \varphi(t) d t \\
\int_{0}^{T}\left(h_{\gamma}\left(u_{\varepsilon m}\right), w\right) \varphi(t) d t \rightarrow \int_{0}^{T}\left(h_{\gamma}(u), w\right) \varphi(t) d t
\end{gathered}
$$

$\forall v \in V, \varphi \in D(0, T)$

Luego la función $u$, satisface:

$$
h_{\alpha}(u)^{\prime}+\chi+h_{\gamma}(u)=0
$$

Finalmente por el método de monotonía se demuestra que:

$$
\chi=A(u)
$$


En efecto, sea

$$
0 \leq X_{\varepsilon m}=\int_{0}^{T}\left\langle A\left(u_{\varepsilon m}\right)-A(v), u_{\varepsilon m}-v\right\rangle d t
$$

Tenemos que:

$$
\begin{array}{r}
\int_{0}^{T}\left\langle A\left(u_{\varepsilon m}\right), u_{\varepsilon m}\right\rangle d t=-\int_{0}^{T}\left(h_{\alpha}\left(u_{\varepsilon m}\right)^{\prime}, u_{\varepsilon m}\right) d t-\varepsilon \int_{0}^{T}\left(u_{\varepsilon m}^{\prime}, u_{\varepsilon m}\right)-\int_{0}^{T}\left(h_{\gamma}\left(u_{\varepsilon m}\right), u_{\varepsilon m}\right) d t \\
=\frac{\alpha+1}{\alpha+2}\left|u_{\varepsilon m}(0)\right|_{\alpha+2}^{\alpha+2}-\frac{\alpha+1}{\alpha+2}\left|u_{\varepsilon m}(T)\right|_{\alpha+2}^{\alpha+2}+\frac{\varepsilon}{2}\left|u_{\varepsilon m}(0)\right|^{2}-\frac{\varepsilon}{2}\left|u_{\varepsilon m}(T)\right|^{2}-\left|u_{\varepsilon m}\right|_{L^{\gamma+2}(Q)}^{\gamma+2}
\end{array}
$$

Entonces

$$
\begin{aligned}
& X_{\varepsilon m}=\frac{\alpha+1}{\alpha+2}\left|u_{\varepsilon m}(0)\right|_{\alpha+2}^{\alpha+2}-\frac{\alpha+1}{\alpha+2}\left|u_{\varepsilon m}(T)\right|_{\alpha+2}^{\alpha+2}- \\
& \quad-\left|u_{\varepsilon m}\right|_{L^{\gamma+2}(Q)}^{\gamma+2}-\int_{0}^{T}\left\langle A\left(u_{\varepsilon m}\right), v\right\rangle d t-\int_{0}^{T}\left\langle A(v), u_{\varepsilon m}-v\right\rangle d t
\end{aligned}
$$

Luego:

$$
\begin{aligned}
0 \leq \limsup X_{\varepsilon m} \leq \frac{\alpha+1}{\alpha+2}\left|u_{0}\right|_{\alpha+2}^{\alpha+2}-\frac{\alpha+1}{\alpha+2}|u(T)|_{\alpha+2}^{\alpha+2}-|u|_{L^{\gamma+2}(Q)}^{\gamma+2}- & \\
& -\int_{0}^{T}\langle\chi, v\rangle d t-\int_{0}^{T}\langle A(v), u-v\rangle d t
\end{aligned}
$$

Por otro lado:

$$
\begin{aligned}
|u(T)|_{\alpha+2}^{\alpha+2}-|u(0)|_{\alpha+2}^{\alpha+2} & =\int_{0}^{T} \frac{d}{d t}|u(T)|_{\alpha+2}^{\alpha+2} d t=\int_{0}^{T} \frac{d}{d t}\left|h_{\alpha / 2}(u(T))\right|^{2} d t \\
& =2 \int_{0}^{T}\left(h_{\alpha / 2}(u(T)), h_{\alpha / 2}(u(T))^{\prime}\right) d t=(\alpha+2) \int_{Q}|u|^{\alpha / 2} u|u|^{\alpha / 2} u^{\prime} d Q \\
& =(\alpha+2) \int_{Q}|u|^{\alpha} u^{\prime} u d Q=\frac{\alpha+2}{\alpha+1} \int_{Q} h_{\alpha}(u)^{\prime} u d Q
\end{aligned}
$$

Luego

$$
\begin{aligned}
\frac{\alpha+1}{\alpha+2}|u(0)|_{\alpha+2}^{\alpha+2}-\frac{\alpha+1}{\alpha+2}|u(T)|_{\alpha+2}^{\alpha+2}- & |u|_{L^{\gamma}+2(\Omega)}^{\gamma+2}= \\
& =-\int_{0}^{T}\left(h_{\alpha}(u)^{\prime}, u\right) d t-\int_{0}^{T}|u(t)|_{\gamma+2}^{\gamma+2} d t=\int_{0}^{T}\langle\chi, u\rangle d t
\end{aligned}
$$

Por lo tanto

$$
0 \leq \int_{0}^{T}\langle A(u)-A(v), u-v\rangle d t, \forall v \in L^{p^{\prime}}\left(0, T ; V^{\prime}\right)
$$

Y tenemos probado que $A u=\chi$.

\section{REFERENCIAS BIBLIOGRÁFICAS}

[1] M. Berger, Nonlinearity and functional analisys, Academic Press. New York-San FranciscoLondon, 1977.

[2] H. Fujita, On Some non Existence and non Uniqueness Theorems for non Linear Parabolic Equations. 
[3] V. Galaktionov, On Unbounded Solutions of Cauchy Problem for the Parabolic Equation $u_{t}=\Delta\left(u^{\rho} \nabla u\right)+u^{1+\beta}$; Dokl-Akad. Nauk. SSRR 252, No6, 1362-1364, 1980.

[4] V. Galaktionov, On Unbounden Solutions of Semilinear Parabolic Equations. Akd.Nauk. SSRR No161, Moscow, 1979.

[5] V. Galaktionov, On a Boundary Value Problem for the non Linear Parabolic Equation $u_{t}=\Delta\left(u^{\rho+1}+u^{\beta}\right)$. Differents. Ur'niya 17, No5, 836-842, 1981.

[6] V. Galaktionov, The Existence and non Existence of Global Solutions of Boundary Value Problems for Quasilinear Parabolic Equations. USSR. Comput. Maths. Phys.Vol. 22, No6, pp 88-107, 1982.

[7] R. Izaguirre Maguiña. Existencia de solucion Global para una clase de Ecuaciones Parabólicas No- Lineales. Revista de la Facultad de Ciencias Matemáticas- 1-Noviembre 1987-UNMSM.

[8] R. Izaguirre Maguiña. Solucoes de Problemas em Equacoes Diferenciais Parciais Degeneradas. IM-UFRJ Tese de Doutor. Decembro - 1986.

[9] O. Ladyzhenskaya, V. Solonnikov, N. Ural'Teva. Linear and Quasi-Linear Equations of Parabolic Type. Nauka, Moscow, 1967.

[10] J.L. Lions, Quelques Methodes de Resolution Des Problemes Aux Limites non Lineaires, Dunod, Gauthier, Villars. Paris, 1969.

[11] P. Raviart, Sur la Resolution de Certaines Equations Parabolic non Lineaires. J. of Func. Anali, 5, 299-329 (1970).

[12] A. Samarskii, Heat Structures and the Fundamental Lenght in a Medium with non-linear heat Conduction and Three-dimensional heat Sources. Dokl. Nauk. SSSR. 227, No2, 321324, 1976.

[13] A. Samarskii, Burning of a non-linear Medium in the form of Composite Structures. Dolk. Akad. Nauk. SSSR. 237, No6, 1330-1333, 1977.

[14] D. Sattinger, On Global Solution of non-linear Hiperbolic Equations. Arch. Rat. Mech. Analysis, 30, 148-172, 1968.

[15] R. Showalter, Monotone Operarators in Banach Space and Non Lineal Partial Differential Equations. Mathematical Surveys and Monograph, Vol. 45, AMS, 1991.

[16] J. Simon, Compact sets in the space $L^{p}(0, T ; B)$. Ann. Mat. Pura Appl. (4), 146:65-96, 1987.

[17] W. Strauss, Evolution Equations non-linear in the Time derivate. J. Math. Mech.15, (1966), 49-82.

[18] M. Tsutsumi. Existence and non-existence of Global Solutions for non-linear Parabolic Equations. Publs. Res. Inst. Math. Scl. 8. 211-229. 1972/1973. 